\title{
A Plug-in Feed-forward Control for Sloshing Suppression in Robotic Teleoperation Tasks
}

\author{
Luigi Biagiotti, Davide Chiaravalli, Lorenzo Moriello, Claudio Melchiorri
}

\begin{abstract}
In this paper, the problem of suppressing sloshing dynamics in liquid handling robotic systems has been faced by designing a dynamic filter that starting from the desired motion of the liquid container calculates the complete position/orientation trajectory for the robot end-effector. Specifically, a design philosophy mixing a filtering technique that suppresses the frequency contributions of the reference motion that may cause liquid oscillations and an active compensation of lateral accelerations by a proper container re-orientation has been adopted. In principle, the latter contribution requires the knowledge of acceleration of the reference trajectory, but because of the use of an harmonic smoother that performs a shaping of the original motion, it is possible to obtain the value of the acceleration in runtime. In this way, the proposed methods can be applied also to reference motions that are not known in advance, e.g. commands directly provided by a human operator. This possibility has been demonstrated by means of a number of experimental tests in which the user teleoperates the robot carrying the container with the liquid by simply moving in the free space its hand, whose 3D position is detected by a motion capture system.
\end{abstract}

\section{INTRODUCTION}

The use of robotic systems in new tasks, traditionally executed by human operators or by means of process control systems, may involve the manipulation of liquid materials. These operations can be particularly critical because of the liquid slosh induced by the container motion, which can determine the spilling of the liquid itself. For instance, the need of moving, manipulating, pouring liquid ingredients in the food processing industry motivates our previous work [1], in which the desired trajectories are filtered with a so-called exponential smoother in order to suppress the oscillations of the liquid that are seen as vibrations in a resonant mechanical system. Afterwards, the algorithm has been improved by combining the trajectory filtering action with a compensation of the lateral acceleration by properly controlling the 3D orientation of the vessel [2]. The proposed approach is a typical example of feed-forward control providing the proper reference trajectory for the robot/machine on the basis of the model of the slosh dynamics. With respect to feedback methods that rely on a measure of the liquid surface configuration, feed-forward algorithms offer some

L. Biagiotti is with the Department of Engineering "Enzo Ferrari", University of Modena and Reggio Emilia, via Pietro Vivarelli 10, 41125 Modena, Italy, e-mail: luigi.biagiotti@unimore.it.

D. Chiaravalli, L. Moriello and C. Melchiorri are with the Department of Electrical, Electronic and Information Engineering "Guglielmo Marconi", University of Bologna, Viale del Risorgimento 2, 40136 Bologna, Italy, e-mail: \{davide.chiaravalli2\},\{lorenzo.moriello2\} ,$\{$ claudio.melchiorri $\} @$ unibo.it.

This activity has been supported by the University of Modena and Reggio Emilia with the "FAR 2017" project. advantages since they do not require an additional sensor apparatus (that in case of detection of the liquid configuration may be quite complicated) and can be easily implemented in standard industrial robots/machines, without modifications of their control systems. For this reason the techniques based on feed-forward are the most common solutions in the field of liquid manipulation. For instance, in [3] and [4] the of slosh dynamics is compensated via tilt angle modification. Input shaping techniques are widely used in conjunction with smooth trajectory planning and other kinds of filtering/smoothing methods, see [5], [6], [7], [8], [9] among many others. Alternative feed-forward methods are based on the optimization of the reference trajectories applied to the liquid container, computed by taking into account the dynamic model of the system and a number of constraints, like maximum velocity, acceleration, etc. [10], [11], [12]. The main drawback of all these methods is obviously the poor robustness of the system with respect to unmodelled dynamics (in this specific case higher order sloshing modes) and inaccurate knowledge of the physical parameters. For this reason, some authors combine the feed-forward control with a feedback compensation, e.g. based on $H_{\infty}$ loop shaping methods [5], [13], or mix different feed-forward algorithms like input shaping and smoothing filters [9], or smoothers and tilting compensation, like in our previous paper [2].

In this paper, an approach that follows the same general idea of [2], i.e. the combination of a filtering action on the reference motion and a compensation of the lateral acceleration by means of a proper orientation of the liquid container, has been developed. The novelty of this method lies in the fact that the proposed feed-forward controller does not require any knowledge of the reference motion but can be used independently from the specific input or the particular robot. In fact, a plug-in component has been implemented that can be used in any robotic application involving the manipulation of liquids by simply inserting it between the motion planner and the robot controller. In order to demonstrate the effectiveness of the proposed methods a simple teleoperation task has been arranged: the user directly commands the robot by moving in the free space its hand, whose $3 \mathrm{D}$ position is detected by a motion capture system, and the robot, carrying a pot filled with water, tracks the motion generated by the devised algorithm.

\section{A SIMPLIFIED APPROACH TO SLOSH-FREE MOTION}

In order to explain the proposed control approach, a simplified scenario, in which the container is moved on a 


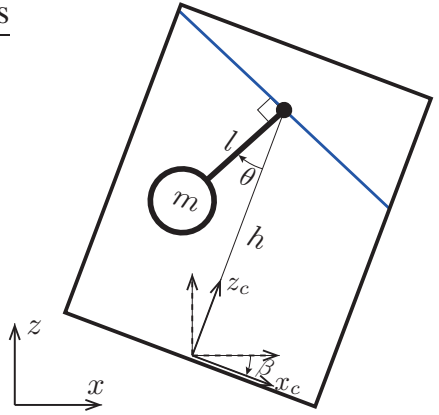

Fig. 1. Simplified mechanical model approximating the first asymmetric sloshing mode in a cylindrical vessel.

plane and rotated about an axis orthogonal to this plane, is initially considered. The dynamics of the liquid in the moving vessel can be modelled with an equivalent mechanical model consisting of a rigid mass $m_{0}$ and a series of pendulums with mass $m_{j}$, length $l_{j}$, and support point located at a distance $L_{j}$ from the undisturbed free surface of the liquid, see [2]. For control purposes, the model can be further simplified by considering only the first asymmetric mode of the slosh, that is a single pendulum with mass $m$, length $l$ and pivot located at the centre of the liquid surface. Moreover, it is assumed that the pendulum is always orthogonal to the liquid surface which is supposed to be flat. Therefore, the model of the sloshing dynamics inside a container moving on the plane can be represented as in Fig. 1. The position of the mass of the pendulum is given by

$$
\begin{aligned}
& x_{m}=x_{c}-h \sin (\beta)+l \sin (\beta+\theta) \\
& z_{m}=z_{c}+h \cos (\beta)-l \cos (\beta+\theta)
\end{aligned}
$$

where the pair $\left(x_{c}, y_{c}\right)$ denotes the position of the container with respect to the base reference frame, $\beta$ is the tilting angle imposed to the container (it is supposed that the center of rotation is located along the centreline at a distance $h$ from the pendulum pivot) and $\theta$ denotes the rotation angle of the pendulum.

The equation of the motion of the pendulum can be derived by using the Lagrange equations. The kinetic energy of the mass $m$ is given by

$$
\begin{aligned}
T= & \frac{1}{2} m\left[\dot{x}_{m}^{2}+\dot{z}_{m}^{2}\right] \\
= & \frac{1}{2} m\left[\left(\dot{x}_{c}-h \cos (\beta) \dot{\beta}+l \cos (\beta+\theta)(\dot{\beta}+\dot{\theta})\right)^{2}\right. \\
& \left.+\left(\dot{z}_{c}-h \sin (\beta) \dot{\beta}+l \sin (\beta+\theta)(\dot{\beta}+\dot{\theta})\right)^{2}\right]
\end{aligned}
$$

while its potential energy is equal to

$$
V=m g z_{m}=m g\left(z_{c}+h \cos (\beta)-l \cos (\beta+\theta)\right)
$$

where $g$ is the gravity acceleration. The Lagrangian can be computed as $\mathcal{L}=T-V$. By considering the Lagrange equation with respect to $\theta$, the differential equation

$$
\begin{gathered}
l \ddot{\theta}+(l-h \cos (\theta)) \ddot{\beta}-h \sin (\theta) \dot{\beta}^{2}+ \\
+\cos (\beta+\theta) \ddot{x}_{c}+\sin (\beta+\theta)\left(g+\ddot{z}_{c}\right)+\frac{b}{m l} \dot{\theta}=0
\end{gathered}
$$

describing the dynamics of the pendulum is obtained. The dissipative term $\frac{b}{m l} \dot{\theta}$ takes into account the damping force between the liquid and the container. Note that (1) is exactly the same equation found in [3], but the control solution that will be deduced to maintain $\theta$ to zero is rather different from the one proposed here.

It is initially assumed that the container cannot be arbitrarily rotated, and accordingly $\ddot{\beta}=\dot{\beta}=\beta=0$. In this way, the motion equation becomes

$$
l \ddot{\theta}+\frac{b}{m l} \dot{\theta}+\cos (\theta) \ddot{x}_{c}+\sin (\theta)\left(g+\ddot{z}_{c}\right)=0
$$

where $\ddot{x}_{c}$ and $\ddot{z}_{c}$ are the external inputs. The equilibrium point of (2) for generic values of the inputs is

$$
\theta=-\arctan \left(\frac{\ddot{x}_{c}}{g+\ddot{z}_{c}}\right)
$$

and it is stable for $-\pi / 2<\theta<\pi / 2$. In this case, the translational motions of the container produce a pendulum swing that depends on the accelerations and tends to the value (3). By linearizing (2) for $\theta=\dot{\theta}=0$ and $\ddot{x}_{c}=\ddot{z}_{c}=0$ the model

$$
l \ddot{\theta}+\frac{b}{m l} \dot{\theta}+g \theta=-\ddot{x}_{c}
$$

can be deduced. This model is equivalent to the general second order equation

$$
\ddot{\theta}+2 \delta \omega_{n} \dot{\theta}+\omega_{n}^{2} \theta=u
$$

where the parameters $\omega_{n}$ and $\delta$ depend on the characteristics of liquid and container, and the input $u$ is proportional to the accelerations along the $x$ axis. As usual for a pendulum

$$
\omega_{n}^{2}=\frac{g}{l}
$$

As mentioned in the introduction, the model (4) suggests that a simple and effective way to reduce the pendulum swing $\theta$ caused by the input $u$ consists in shaping such an input by means of a proper filter, according to a typical approach for residual vibration suppression in mechanical systems.

If now the rotation $\beta$ is considered, by assuming the initial condition $\theta=\theta_{0}=0$ in the motion equation (1) the term $h \sin (\theta) \dot{\beta}^{2}$ becomes negligible. Moreover if $h=l$ (that is the center of rotation of the container is located at the rest position of the pendulum) $(l-h \cos (\theta)) \ddot{\beta} \approx 0$, and the equation (1) becomes

$$
l \ddot{\theta}+\frac{b}{m l} \dot{\theta}=-\cos (\beta) \ddot{x}_{c}-\sin (\beta)\left(g+\ddot{z}_{c}\right) .
$$

By acting on $\beta$, it is possible to enforce $\ddot{\theta}=\dot{\theta}=0$, and therefore $\theta \equiv 0$, imposing that the second member of (6) is zero. To this purpose, the angle $\beta$ must be chosen as

$$
\beta=-\arctan \left(\frac{\ddot{x}_{c}}{g+\ddot{z}_{c}}\right) .
$$

Therefore, it is possible to conclude that an effective strategy to avoid sloshing consists in rotating the liquid container of an angle that equals the equilibrium angle of the virtual pendulum without compensation (compare equations (7) and (3)). Differently from [3] and [2], in which the center of rotation of the container coincides with pendulum pivot, 
it is convenient to locate it in the rest position of the pendulum mass. In this way the angular acceleration $\ddot{\beta}$ does not influence the pendulum dynamics.

\section{3D SLOSHING SUPPRESSION}

The generalization to the 3D case of the control approach devised in previous section immediately descends from the considerations related to the planar case. Initially, the reference position trajectory $p(t)=[x(t), y(t), z(t)]^{T}$ must be filtered in order to suppress the residual vibration due to sloshing mode characterized by $\left(\delta, \omega_{n}\right)$. In particular, the so called damped harmonic smoother is adopted [14], i.e. a dynamic filter whose analytical expression can be expressed in the Laplace domain as

$$
H(s)=\frac{\sigma^{2}+\left(\frac{\pi}{T}\right)^{2}}{1+e^{\sigma T}} \frac{1+e^{-s T} e^{\sigma T}}{(s-\sigma)^{2}+\left(\frac{\pi}{T}\right)^{2}}
$$

where $\sigma$ and $T$ are constant parameters that can be freely chosen. In particular, $T$ represents the duration of the impulse response of the filter and therefore the additional delay produced by the smoother on the filtered signal with respect to its original duration. The smoother is characterized by the pole-zero map of Fig. 2. The cancellation of the oscillating dynamics described by (4) can be obtained by assuming

$$
\sigma=-\delta \omega_{n}, \quad T=\frac{3}{2} \frac{2 \pi}{\omega_{n} \sqrt{1-\delta^{2}}} .
$$

Concerning the problem of vibration suppression the smoother behaves like an input shaper, but with respect to a basic Zero Vibration (ZV) input shaper it causes a delay of triple value. However, some features of the harmonic smoother make it the ideal filter for this application:

- The smoother, composed by a two-impulse input shaper and a second order low-pass filter, besides reducing the vibration caused by the filtered signal, increases its level of smoothness of two. In this manner, the output of the filter is at least of class $\mathcal{C}^{2}$, i.e. with continuous acceleration, provided that the input signal is continuous.

- The structure of the filter expressed in the controllable canonical form, reported in Fig. 3, allows to obtain not only the filtered output but also its first and second derivatives.

- Because of the low-pass characteristics of its frequency

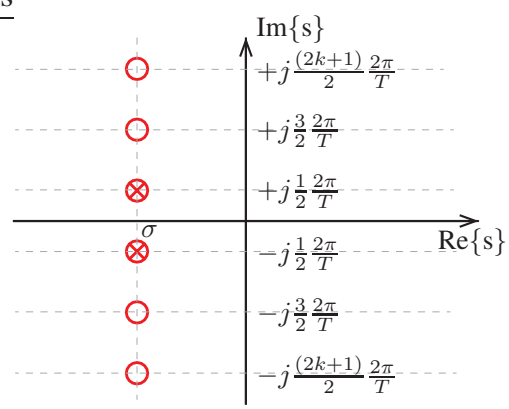

Fig. 2. Pole-zero map of the damped harmonic smoother $H(s): \sigma$ and $T$ are the free parameters that appear in (8).

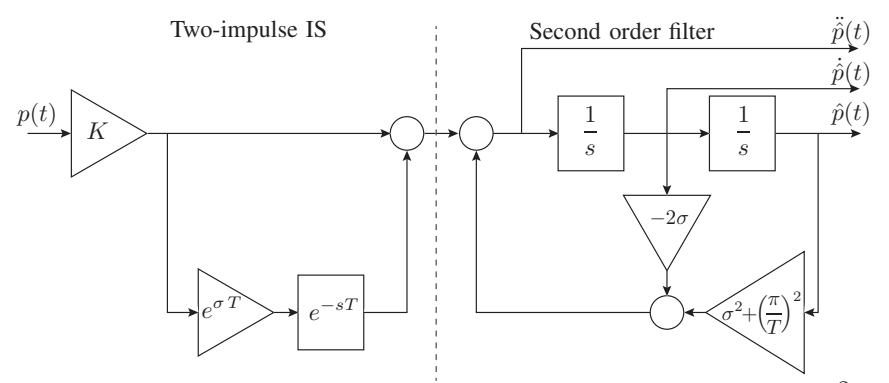

Fig. 3. Harmonic smoother structure with constant $K=\frac{\sigma^{2}+\left(\frac{\pi}{T}\right)^{2}}{1+e^{\sigma T}}$.

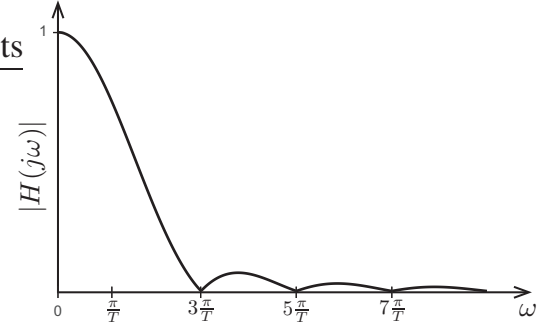

Fig. 4. Magnitude of the frequency response of the damped sinusoidal filter $H(s)$ with $\sigma=-0.0431$ and $T=0.7211 \mathrm{~s}$.

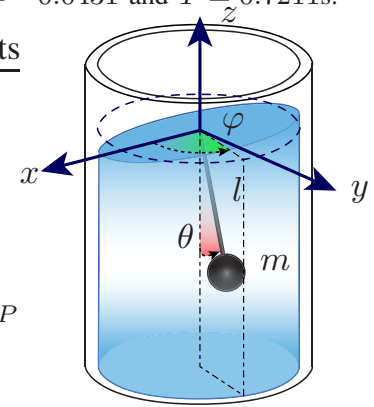

Fig. 5. Spherical pendulum modelling the sloshing dynamics in a liquidfilled vessel that moves along a 3D trajectory.

response, shown in Fig. 4, the smoother permits the suppression of higher sloshing modes. Note that $|H(j \omega)|$ is proportional to the PRV (Percent Residual Vibration) induced by filtered signal when the resonant plant is completely undamped and its resonant frequency is affected by an estimation errors, see [14] for more details. As a consequence $|H(j \omega)|$ provides a measure of the robustness of the filter, and, therefore, it clearly shows that additional sloshing modes characterized by $\omega_{n}>3 \frac{\pi}{T}$ are effectively reduced.

The filtered trajectory $\hat{p}(t)=[\hat{x}(t), \hat{y}(t), \hat{z}(t)]^{T}$ is used for constructing the orientation trajectory of the robot manipulator with the purpose of aligning the container with the (equilibrium) angular position of the virtual pendulum caused by the acceleration $\ddot{\hat{p}}(t)$. As shown in Fig. 5, the spherical pendulum configuration can be fully described by means of the angles $(\theta, \varphi)$. In [2] the dependance of these angles from the linear acceleration imposed to the vessel has been analytically deduced, i.e.

$$
\begin{aligned}
& \theta=\tan ^{-1}\left(\frac{\sqrt{\tilde{\hat{x}}^{2}+\ddot{\hat{y}}^{2}}}{g+\ddot{\hat{z}}}\right) \\
& \varphi=\pi+\operatorname{atan} 2(\ddot{\hat{y}}, \ddot{\hat{x}})
\end{aligned}
$$

where atan2 is the four quadrant inverse tangent. The robot manipulator must rotate the vessel about the point where the 


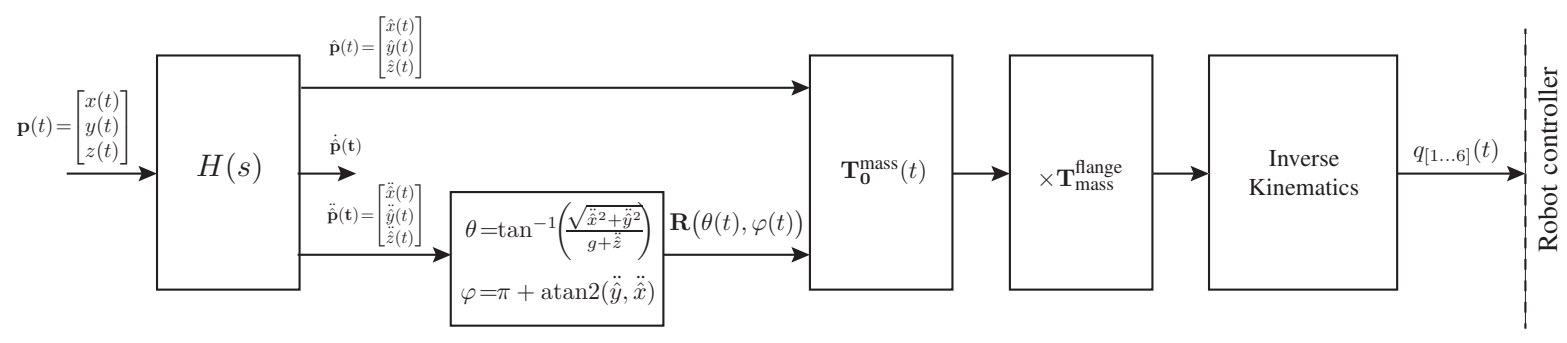

Fig. 6. Feed-forward controller for sloshing compensation based on the harmonic smoother.

mass $m$ is located at rest in order to impose the orientation given by $(\theta, \varphi)$. Therefore, two consecutive rotations $\operatorname{Rot}_{z}(\varphi)$ and $\operatorname{Rot}_{y}(-\theta)$ must be applied. Moreover, since the first rotation about the $z$ axis would cause an undesired vessel rotation of an angle $\varphi$ about its symmetry axis, it is necessary to add the converse rotation about the $z$ axis after $\operatorname{Rot}_{y}(-\theta)$. Finally, the desired configuration of the vessel is

$$
\mathbf{R}(\theta, \varphi)=\operatorname{Rot}_{z}(\varphi) \operatorname{Rot}_{y}(-\theta) \operatorname{Rot}_{z}(-\varphi) .
$$

It is worth to noticing that the term $\operatorname{Rot}_{z}(-\varphi)$ offers an additional advantage when $\ddot{\hat{y}}=\ddot{\hat{x}}=0$. In this case, the angle $\varphi$ in (11) is not well defined; however, since $\theta=0$, $\mathbf{R}(\theta, \varphi)=\operatorname{Rot}_{z}(\varphi) \operatorname{Rot}_{z}(-\varphi)=I_{3}$, being $I_{3}$ the $3-$ by -3 identity matrix.

By considering the expressions of $\theta$ and $\varphi$ that appear in (10) and (11) and are used to define the instant value of the rotation matrix $\mathbf{R}(\theta, \varphi)$, the benefits of the harmonic smoother, that guarantees the continuity of the accelerations for any kind of input (supposed continuous) and allows their computation online, are evident.

The complete structure of the proposed feed-forward controller for robust sloshing compensation with unknown reference signal is illustrated in Fig. 6. The filtered trajectory $\hat{\mathbf{p}}(t)$ and the rotation matrix $\mathbf{R}(\theta(t), \varphi(t))$ are arranged in the homogeneous transformation matrix

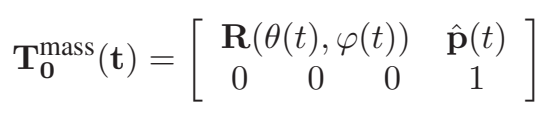

that provides the desired configuration of the reference frame attached to container with respect to the world reference frame. In order to take into account the relative pose of the container with respect to the robot flange, matrix $\mathbf{T}_{0}^{\text {mass }}(\mathbf{t})$ is multiplied by the constant matrix $\mathbf{T}_{\text {mass }}^{\text {flange }}$, i.e.

$$
\mathbf{T}_{0}^{\mathrm{flange}}(\mathbf{t})=\mathbf{T}_{\mathbf{0}}^{\text {mass }}(\mathbf{t}) \cdot \mathbf{T}_{\text {mass }}^{\text {flange }}
$$

Finally, the instant configuration of the robot flange $\mathbf{T}_{0}^{\text {flange }}(\mathbf{t})$ is processed using the inverse kinematics to derive the joint trajectories $q_{[1 \ldots 6]}(t)$ that the robot manipulator must track.

\section{EXPERIMENTAL TESTS}

The method discussed in the previous two sections has been tested by means of the experimental setup shown in Fig. 7. The system has been designed according to a simple master-slave teleoperation architecture, in which the human operator directly commands the position of the robot, carry- ing the container with the liquid, by moving a hand in the 3D space. As a consequence, the use of the proposed feedforward filter implements a sort of shared control because the user does not take care of the sloshing while the control system provides a compensation of this phenomenon.

From the hardware point of view, on the master side the reference trajectory produced by the motion of the operator hand is tracked by a Vicon Motion capture system. The Vicon cameras (Bonita 10), operating at their maximum framerate $(250 \mathrm{fps})$ with a $2 \mathrm{~ms}$ latency, are connected to the main workstation running the Robotic Operating System (ROS) Kinetic distribution on Ubuntu 16.04. The acquired trajectory is filtered and then sent to the slave side where a steel pot (of radius $97.5 \mathrm{~mm}$ ), filled with 3 liters of water, has been connected to the flange of a Comau Smart5 Six anthropomorphic robot. A pc running the RTAI-Linux 3.9 operating system on a Ubuntu NATTY distribution with Linux kernel 2.6.38.8. allows the communication between the main workstation and the robot controller thanks to a ROS wrapper, defined using the "C4G OPEN" software functionality of the Comau controller.

In order to monitor the liquid surface during the tests an ASUS Xtion PRO Live RGB-D camera has been mounted on a specifically-designed support above the container. A blue pigment has been added to the water to make the detection easier. A second RGB-D camera has been installed on the top of the manipulator third joint in order to provide a visual feedback of the robot movements to the user.

Note that the motions of the human operator are used as reference trajectories without any kind of modification or scaling operation. The only limitation imposed to the task concerns the robot workspace, that, for safety reasons, has been restricted to a box of $0.6 \times 1 \times 0.8$ meters (along $x$, $y$ and $z$ directions, respectively) centered around the initial position.

\section{A. Test results}

The proposed feed-forward methods relies on the correct estimation of some parameters of the system. In particular, the harmonic smoother requires the knowledge of the natural frequency and damping ratio related to the sloshing to be inserted in (9). These values have been experimentally deduced in [2] and are equal to $\omega_{n}=13.076 \mathrm{rad} / \mathrm{s}, \delta=$ 0.005 . The orientation compensation is only based on the translational accelerations with the center of rotation located along the symmetry axis of the cylindrical container at a 


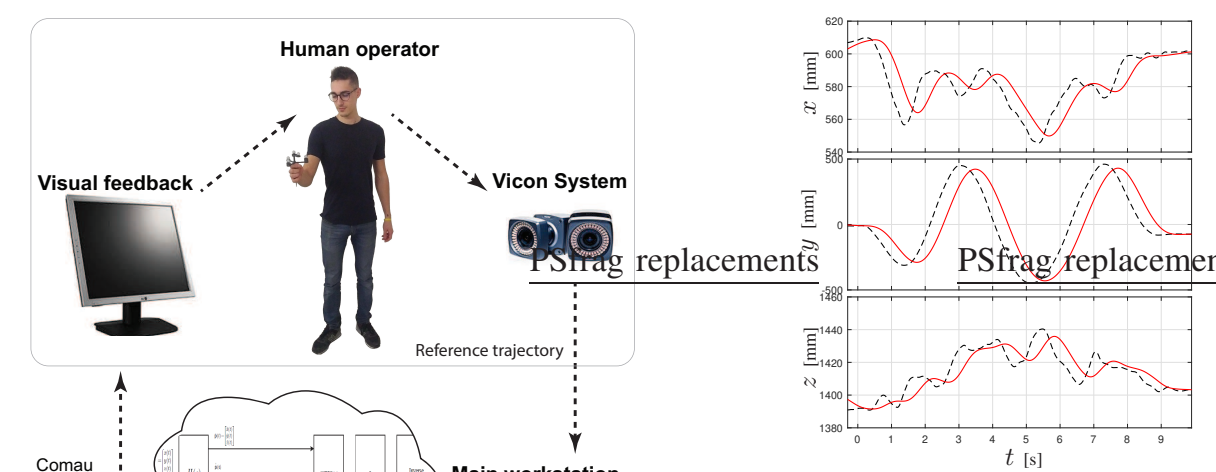

(a)

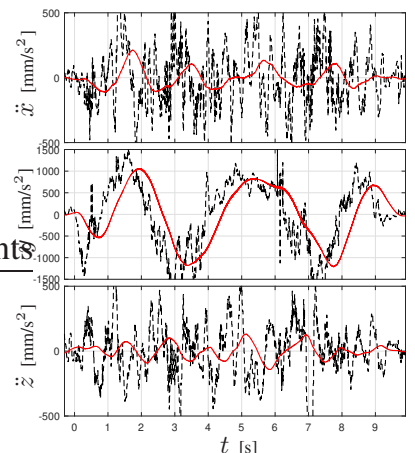

(b)

Fig. 9. Motion profiles corresponding to the geometric path of Fig. 8: positions (a) and accelerations (b). The black dashed lines are related to the original motion while the red solid lines represent the filtered trajectory.

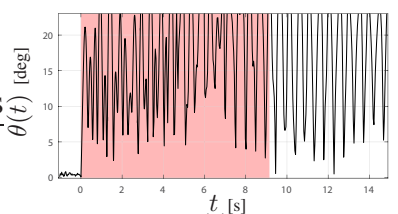

(a)

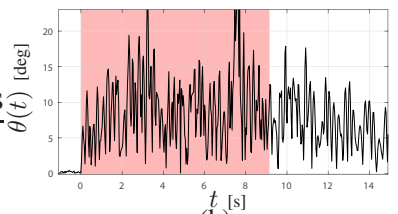

(b)

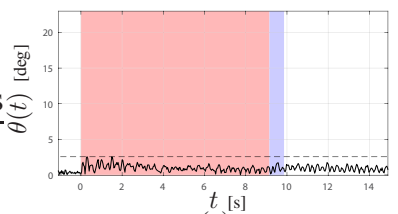

(c)

Fig. 10. Sloshing angle profiles measured when the liquid vessel is moved according to the trajectories of Fig. 9: unfiltered trajectory (a); unfiltered trajectory with orientation compensation (b); smoothed trajectory with orientation compensation (c). The red area indicates the motion time while the blue area highlights the time lag $T$ introduced by the filter.

is reported: it is a simple linear movement along the $y$ axis performed by the user several times. The corresponding motion profiles as a function of time are shown in Fig. 9. The black (dashed) lines are related to the original motion while the red (solid) lines represent the trajectory filtered by the harmonic smoother. This notation is used throughout this section. Beside the position profiles also the acceleration profiles are reported. In the unfiltered case, the accelerations are obtained via numerical differentiation. This procedure amplifies the noise due to the measurement process and to the tremors and small movements that unavoidably affect the human user and leads to very high peak values. On the other hand, the filtered trajectory is characterized by very smooth profiles of both position and acceleration but with respect to the original motion it introduces a delay that, with the above values of the sloshing parameters, is $T=0.6895 \mathrm{~s}$.

The effect of the proposed technique on the sloshing dynamics are illustrated in Fig. 10, showing the tilting angle $\theta$ of the liquid surface obtained in different conditions when the linear trajectory in Fig. 9 is considered. It is worth to noticing that the measure of the angle $\theta$ is obtained from the In Fig. 9 the geometric path of the first standard motion 


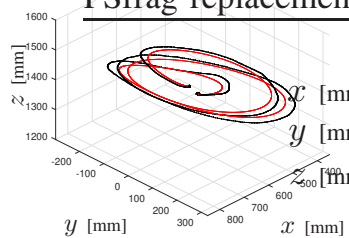

(a)

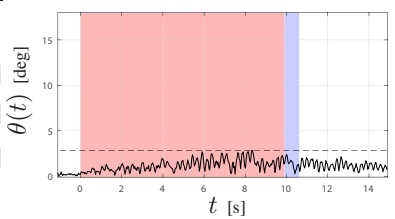

(b)
Fig. 11. Geometric paths disposed on the plane $x-y$ used to test the proposed algorithm (a) and resulting sloshing angle $\theta$ (b).

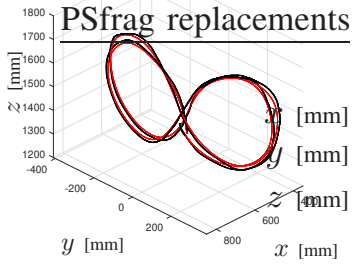

(a)

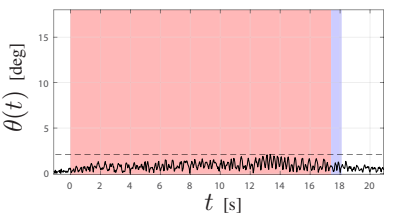

(b)
Fig. 12. Geometric paths disposed on the plane $y-z$ used to test the proposed algorithm (a) and resulting sloshing angle $\theta$ (b).

images acquired with the RGB-D camera, by approximating the liquid surface with a plane and then computing the inclination angle by a coordinate transformation. For more details on this procedure see [2]. In Fig. 10(a) it is shown the sloshing behavior caused by the acquired trajectory of Fig. 9(a) without any compensation. Despite the motion profile in Fig. 9(a) appears to be quite smooth, its application to the liquid container triggers a huge sloshing dynamics that causes the spilling of the liquid.

The behavior seems improved in Fig. 10(b), where the result consequent to the application of same trajectory with the compensation of the orientation is shown. Indeed, a direct inspection of the liquid motion reveals that in this case the sloshing level is not reduced. As a matter of fact, a sort of chaotic behavior starts on the liquid surface probably caused by the noisy acceleration profile (see Fig. 9(b)) that excites high order sloshing modes via the angular compensation. In this case, the hypothesis of planar surface of the liquid is not valid and, accordingly, the measurement of $\theta$, based on the planar interpolation of the point provided by the RGB$\mathrm{D}$ camera, fails. However, in the accompanying video, it is possible to appreciate the real sloshing dynamics. When finally the harmonic smoother (together with the orientation compensation) is inserted between the original trajectory and the robot, the range of variation of the sloshing angle $\theta$ is drastically reduced both during the trajectory and at the end of the motion, see Fig. 10(c). The price for maintain the angle $\theta$ below 2.5 degrees is the delay $T$ and a slight deformation of the geometric path. Similar results are obtained when the user performs motions on the horizontal plane $x-y$, see Fig. 11, or in the vertical plane $y-z$, see Fig. 12 .

Further experiments have been performed online. In this case the user provides casual and very long motion trajectories to the robot. For the sake of clarity, the results are not reported here since the resulting paths are very confused and the profile of the sloshing angle $\theta$ not very meaningful.
The interested reader can find one of these experiments in the accompanying video. The motion, with an approximate duration of one minute and a maximum acceleration of 1 $\mathrm{m} / \mathrm{s}^{2}$, causes a sloshing angle not exceeding 8 degrees.

\section{CONCLUSIONS}

In this paper, the feed-forward method proposed in [2] for sloshing suppression in robotic applications and based on a filtering action combined with a tilting compensation algorithm that counteracts the robot lateral accelerations has been enhanced by eliminating the hypothesis of a priori knowledge of the reference trajectory. In this way, the algorithm has became independent from the particular trajectory generator, that in the paper is a human operator.

The key point of the proposed solution is the so-called harmonic smoother, that not only shapes the input trajectory so that the sloshing at the end of motion is strongly mitigated, but also provides the acceleration of the filtered signal.

The extensive experimental activity demonstrates the effectiveness of the proposed method, that can be profitably used in all the manipulation tasks of liquids involving robots able to control position and orientation of the vessel.

\section{REFERENCES}

[1] L. Moriello, L. Biagiotti, C. Melchiorri, and A. Paoli, "Control of liquid handling robotic systems: A feed-forward approach to suppress sloshing," in Robotics and Automation (ICRA), 2017 IEEE International Conference on, Singapore, May 2017.

[2] —_ "Manipulating liquids with robots: A sloshing-free solution," Control Engineering Practice, vol. 78, pp. 129 - 141, 2018.

[3] J. T. Feddema, C. R. Dohrmann, G. G. Parker, R. D. Robinett, V. J. Romero, and D. J. Schmitt, "Control for slosh-free motion of an open container," IEEE Control Systems, vol. 17, no. 1, pp. 29-36, Feb 1997.

[4] S. J. Chen, B. Hein, and H. Worn, "Using acceleration compensation to reduce liquid surface oscillation during a high speed transfer," in Proceedings 2007 IEEE International Conference on Robotics and Automation, April 2007, pp. 2951-2956.

[5] K. Terashima, M. Hamaguchi, and K. Yamaura, "Modeling and input shaping control of liquid vibration for an automatic pouring system," in Decision and Control, 1996., Proceedings of the 35th IEEE Conference on, vol. 4, Dec 1996, pp. 4844-4850.

[6] A. Aboel-Hassan, M. Arafa, and A. Nassef, "Design and optimization of input shapers for liquid slosh suppression," Journal of Sound and Vibration, vol. 320, no. 1, pp. 1 - 15, 2009.

[7] B. Pridgen, K. Bai, and W. Singhose, "Shaping container motion for multimode and robust slosh suppression," Journal of Spacecraft and Rockets, vol. 50, no. 2, pp. 440-448, Mar 2013.

[8] W. Aribowo, T. Yamashita, and K. Terashima, "Integrated trajectory planning and sloshing suppression for three-dimensional motion of liquid container transfer robot arm," Journal of Robotics, pp. 1 - 15, Jan 2015.

[9] Q. Zang, J. Huang, and Z. Liang, "Slosh suppression for infinite modes in a moving liquid container," IEEE/ASME Transactions on Mechatronics, vol. 20, no. 1, pp. 217-225, Feb 2015.

[10] K. Yano and K. Terashima, "Robust liquid container transfer control for complete sloshing suppression," IEEE Transactions on Control Systems Technology, vol. 9, no. 3, pp. 483-493, May 2001.

[11] _ - "Sloshing suppression control of liquid transfer systems considering a 3-d transfer path," IEEE/ASME Transactions on Mechatronics, vol. 10, no. 1, pp. 8-16, Feb 2005.

[12] L. Consolini, A. Costalunga, A. Piazzi, and M. Vezzosi, "Minimumtime feedforward control of an open liquid container," in IECON 2013 - 39th Annual Conf. of the IEEE, Nov 2013, pp. 3592-3597.

[13] K. Terashima and K. Yano, "Sloshing analysis and suppression control of tilting-type automatic pouring machine," Control Engineering Practice, vol. 9, no. 6, pp. $607-620,2001$

[14] L. Biagiotti, C. Melchiorri, and L. Moriello, "Damped harmonic smoother for trajectory planning and vibration suppression," submitted to IEEE Transactions on Control Systems Technology. 